Artículo de Investigación

A puntes del CENES

ISSN 0120-3053

Volumen 32 - №. 56

Julio - Diciembre de 2013

Págs. 205-236

\title{
Teoría del Pecking Order versus teoría del Trade off para la empresa Coservicios S.A. E.S.P.
}

\section{Theory of the Pecking Order versus theory of the Trade Off for the company Coservicios S.A. E.S.P.*}

Sandra M ilena Zambrano Vargas** Gustavo Adolfo Acuña Corredor***

Fecha de recepción: 24 de abril de 2013

Nueva versión: 15 de mayo de 2013

Fecha de aprobación: 29 de mayo de 2013

El presente artículo es de investigación científica y tecnológica que surge como resultado del trabajo final de maestría en Administración de la Universidad Nacional de Colombia.

** Ingeniera Industrial de la UPTC, Especialista en Gerencia del Talento Humano de la UPTC, Magíster en Administración de la Universidad Nacional de Colombia, docente de la especialización en Gerencia de Proyectos de la Universidad de Boyacá y docente de las especializaciones en Finanzas y Gerencia del Talento Humano de la UPTC. Tunja, Colombia. Correo electrónico: Sandizambrano@hotmail.com

*** Administrador de empresas, de la Universidad Nacional de Colombia; magíster en Administración, de la Universidad de los Andes; profesor asociado de la Universidad Nacional de Colombia. Bogotá, Colombia. Correo electrónico: gaacunac@unal.edu.co 
Teoría del Pecking O rder versusteoría del Trade off para la empresa Coservicios Sandra M ilena Zambrano V argas - Gustavo A dolfo A cuña Corredor

\section{Resumen}

El presente trabajo pretende analizar el grado de aplicación de dos teorías de estructura de capital que han sido contradictorias y extensamente comparadas. Para la aplicación de la teoría del Trade Off se utilizan dos modelos, el primero propuesto por López y de Luna (2002) y el segundo es el propuesto por Cruz et al., (2003). Para la aplicación de la teoría del Pecking Order se analiza la forma como han sido manejadas las reservas, la deuda a largo plazo, el crecimiento de los activos operacionales netos, la tendencia que ha tenido la rentabilidad operacional de los activos y el EBITDA. Al final se encuentra que en los períodos analizados la teoría más usada ha sido la de Pecking Order la cual ha tenido más evidencia empírica en otras organizaciones, y de la teoría del Trade Off no se encontró aplicación.

Palabras clave: estructura de capital, Trade Off, Pecking Order, nivel de endeudamiento, costo de capital.

Clasificación JEL: M 10, F30, M20, G32, G33, M21

\section{Abstract}

This paper aims to analyze the degree of application of two theories of capital structure that have been contradictory and extensively compared. For the implementation of the Trade Off theory are used two models, the first proposed by Lopez and de Luna (2002) and the second model to be applied is that proposed by Cruz et al. (2003). For the implementation of the Pecking Order theory is analyzed how stocks have been managed, longterm debt, the growth of net operating assets, the trend that has been operating profitability of assets and EBITDA. At the end you will find that in the periods analyzed the most used theory has been to Pecking Order which has been more empirical evidence in other organizations and that Trade Off theory application was not found.

Keywords: capital structure,trade off, pecking order, debt level, cost of capital.

JEL Clasificaction: M 10, F30, M 20, G32, G33, M 21 


\section{INTRODUCCIÓN}

Desde la década del cincuenta del siglo pasado, el tema de la estructura óptima de capital ha sido motivo de intensos debates en lo que se refiere a la mejor forma o en general a la mezcla entre deuda y financiación propia que minimice el costo de capital y a su vez maximice el valor de la firma. Los primeros estudios supusieron mercados de capitales perfectos, luego en la búsqueda de la mejor forma de determinar la mezcla se empezaron a tener en cuenta las imperfecciones del mercado, de las cuales han surgido diversas teorías y un sinnúmero de estudios que buscan validar sus supuestos.

Sin duda alguna dos de las teorías más reconocidas son la teoría del Trade Off y la del Pecking Order, las cuales han servido para que muchos académicos las comparen y midan su grado de aplicación en las organizaciones. Para Shyam y Myers (1994), existe una combinación óptima entre la deuda y el patrimonio que hacen que se maximice el valor de la firma, de manera que la empresa se endeudará hasta un punto en donde el valor marginal del escudo de impuestos en deuda adicional se ve disminuido por el valor presente de posibles costos de aflicción financieros. Algunos estudios realizados por Warner (1977), Shapiro (1991), Kim (1978), Mackie-Mason (1990) y Moreno (1985) analizan el impacto de la deuda y el riesgo de impago de la misma que lleva a tener una mayor posibilidad de quiebra que sucede cuando la empresa no ha gestionado de forma adecuada su actividad.

En el presente artículo se pretende mostrar evidencia de la aplicación de las dos teorías de estructura de capital ya 
expuestas en una empresa de servicios públicos y de esta manera llegar a conclusiones sobre cuál de las dos teorías tuvo una mayor aplicación en los períodos estudiados a partir de la aplicación de modelos propuestos por diferentes autores.

\section{Teoría del Trade Off}

Dentro de lo realizado por algunos estudiosos del tema, se puede citar lo propuesto por Bradley, Harrell y Kim (1984), en donde se habla de la existencia de una estructura óptima cuando se equilibra la ventaja de la deuda con el valor de los costos de quiebra. Miller (1977) afirma que la ventaja fiscal de la financiación de la deuda a nivel de la firma se compensa por la desventaja fiscal de la deuda a nivel personal. Entre tanto, De Angelo y Masulis (1980) advierten sobre la existencia de otros ahorros fiscales diferentes a la deuda, tales como la depreciación contable, las reservas por agotamiento y los créditos tributarios a la inversión. En el modelo propuesto por Jensen y Meckling (1976) se habla de la existencia de una estructura óptima en el momento en que se compensan los costos de la deuda con los beneficios de la misma. Por otro lado, Harris y Raviv (1990) estudian el conflicto que surge entre los directivos de la empresa y sus dueños, de manera que se sepa en qué momento se debe liquidar la empresa cuando se presenta la mejor opción para los accionistas pero no para el gerente ya que los accionistas tienen acceso a la información y a supervisar la gestión. Myers (1984) analiza aspectos como los costos de ajuste, la deuda y los impuestos y los costos de las dificultades financieras (costos de quiebra, los costos de agencia, el riesgo moral y los costos de contratación) y sus implicaciones en la determinación de la estructura óptima de capital en la empresa. Por último Zambrano y Acuña (2011, p.94) “a pesar de que la teoría del Trade Off es acertada al explicar la estructura de capital entre sectores y aquellas empresas que estarían más propensas a ser adquiridas con deuda, aún no se da una explicación de por qué existen muchos ejemplos de firmas con altas rentabilidades que no usan su capacidad de deuda o por qué en países en donde se han reducido los impuestos o el sistema impositivo reduce la ventaja fiscal por deuda, el endeudamiento sigue siendo alto".

\section{Teoría del Pecking Order}

La teoría del Pecking Order basa sus supuestos afirmando que no existe una estructura óptima de capital sino que más bien los gerentes usan la jerarquía de preferencias a la hora de realizar nuevas inversiones, es decir primero se recurre al financiamiento con fondos internos debido a que en ésta no existe asimetría de la información, luego se recurre al endeudamiento y como última alternativa a la emisión de acciones. Al respecto Zambrano y Acuña (2011. p.95) afirman: "El Pecking Order hoy día tiene gran aceptación ya que hay muchas organizaciones de nuestro medio que no buscan la combinación óptima entre 
deuda y capital sino que más bien tratan en todo momento de financiar sus nuevos proyectos con recursos propios". En la actualidad esta teoría logra gran importancia dentro de las organizaciones debido a que en su mayoría las empresas no están en la búsqueda de una combinación óptima sino que más bien tratan de financiar sus nuevas inversiones con recursos propios debido a la aversión que tienen a la asimetría de información en el mercado de capitales.

Entre los estudios más reconocidos sobre el tema están los de Myers (1984), quien es reconocido como el principal exponente de la teoría de la jerarquía de preferencias y quien afirma que las empresas prefieren financiarse con recursos internos mediante la reinversión de las utilidades para aprovechar las oportunidades de inversión en el mercado. También sostiene que las empresas cuando requieren recursos externos prefieren hacer uso de la deuda y cuando esta posibilidad se agota, se escoge la emisión de bonos. Para Myers no existe una estructura de capital óptima ya que el capital tanto interno como externo se encuentra en los niveles alto y bajo en una escala de jerarquía dentro de la empresa. Por otro lado, Myers y Majluf (1984) dicen que la estructura financiera tiene como objetivo reducir las ineficiencias en las decisiones de inversión causadas por la asimetría de la información. En otro estudio propuesto por Shyam y Myers (1994) se resalta la importancia de la teoría que utiliza una jerarquía de preferencia para el uso de recursos cuando hay déficit interno que obliga a la empresa a acceder a recursos externos.

\section{Estudios en empresas de servicios públicos}

Para el caso de la estructura de capital, algunos estudiosos se han interesado por buscar evidencias en empresas reguladas con el fin de entender la relación entre dicha regulación y la aplicación de alguna teoría por parte de sus directivos, algunos ejemplos de estos estudios son: el realizado por Averchy Leland (1962), quienes analizaron los perjuicios que le generaba a una empresa la regulación; por otro lado Robichek (1978) orienta su investigación en las tasas de retorno sobre el patrimonio y sobre la deuda relacionados con la regulación. Besanko y Spulbert (1992) estudian el problema de la regulación como un conflicto de intereses en donde el precio regulado es flexible y el regulador tiene incentivos para cubrir los gastos de funcionamiento que no necesariamente cubriría las inversiones realizadas. Spulbert y Spiegel (1994) siguen un juego de tres etapas, en primer lugar la empresa regulada decide sobre su nivel de inversión y de endeudamiento, un mercado competitivo establece el valor de mercado de la deuda y del patrimonio y finalmente el regulador establece el valor de la firma a partir de la determinación de las tarifas. Por último Sarmiento (2006) en sus estudios analiza la relación entre la regulación económica y su incidencia dentro de las estructuras de capital de las empresas de servicios públicos. 
Teoría del Pecking O rder versusteoría del Trade off para la empresa Coservicios Sandra M ilena Zambrano V argas - Gustavo A dolfo A cuña Corredor

\section{METODOLOGÍA}

Para el presente estudio se analiza una firma que representa un sector de economía mixta en donde debe haber un balance entre el bienestar general de la comunidad y los intereses propios de los socios de la empresa. Se tuvieron en cuenta los años 2007, 2008, 2009, 2010 y 2011 debido a que son los períodos más recientes y permiten conocer la situación real de la empresa.

Para la realización del estudio se hizo una división en tres partes. En primer lugar se analizó la estructura de capital actual de la empresa. Para encontrar el costo del patrimonio se utilizó el método del CAPM que requiere de información como la tasa libre de riesgo, la tasa de interés del mercado, el riesgo país, los betas del sector, la inflación interna y de Estados Unidos, entre otros.

En segundo lugar se analiza la estructura de capital de la firma a la luz de la teoría del Trade Off, para tal efecto se usan dos modelos,

- El primer modelo propuesto por López y de Luna (2002) parte del supuesto de que el apalancamiento financiero bien gestionado es una fuente de valor para la firma, de ésta forma en la medida que la rentabilidad de los activos se mantenga constante, emplear más deuda en la estructura de financiación incrementa el valor de la empresa, siempre y cuando el costo de capital sea inferior a la rentabilidad de dichos activos.

El fundamento teórico del modelo se centra en la solución propuesta por Modigliani y Miller (1963) y el CAPM (Capital Asset PricingModel) partiendo de una serie de hipótesis restrictivas como son:

1. Las empresas solo emiten dos tipos de activos: acciones y deuda sin riesgo.

2. Los flujos de caja son flujos perpetuos sin crecimiento.

3. No existen costos de agencia ni de quiebra.

4. En su versión inicial no existen impuestos.

- El segundo modelo para aplicar es el propuesto por Cruz et al. (2003). Este modelo sigue teóricamente los mismos planteamientos del modelo de López y De Luna (2002), pero adicionalmente plantea unos escenarios de la economía junto con su probabilidad de ocurrencia, luego se calcula el valor de la utilidad operacional a partir de dichos escenarios y de diferentes niveles de endeudamiento, finalmente se calcula el costo promedio ponderado de capital con los diferentes niveles de deuda y se compara con la utilidad por acción, allí debe encontrarse que el costo de capital más bajo debe generar la utilidad por acción más alta. La teoría nos dice que en ese punto el nivel de endeudamiento es 
el óptimo para la estructura de capital. El modelo utilizado por Cruz et al. (2003) muestra deficiencias debido a que existe un alto grado de subjetividad en el establecimiento de la probabilidad de ocurrencia de los diferentes escenarios de la economía (mala, regular, aceptable, buena, excelente). Por otro lado también debe estimarse la utilidad operacional de acuerdo con dichos escenarios y con diferentes niveles de endeudamiento, lo que también presenta alto juicio del investigador en su cálculo.

Para la aplicación de los dos modelos discutidos se utilizó el método CAPM ajustado para el cálculo del patrimonio y que según Vélez-Pareja (2004), citado en Acuña (2010), es uno de los más aceptados para el cálculo del costo del patrimonio debido a que analiza la firma teniendo en cuenta características del mercado para empresas que no cotizan en bolsa.

En tercer lugar se analiza la estructura de capital desde la teoría del Pecking
Order, en esta se estudian las siguientes variables: como variable dependiente el nivel de endeudamiento y como variables independientes la reinversión de utilidades, el crecimiento de los activos operacionales netos, la rentabilidad operativa de los activos y el EBITDA. En su estudio, Jaramillo (2009) analiza las mismas variables aquí planteadas llegando a conclusiones interesantes sobre el sector de la construcción.

Al final se encuentran conclusiones y recomendaciones para la empresa sobre el grado de implementación de las dos teorías en el período estudiado.

\section{RESULTADOS}

En primer lugar es importante conocer la situación actual de la empresa, mediante la búsqueda de algunos valores relevantes para el estudio, como son:

\section{Comportamiento de la estructura de capital}

La estructura de capital de la firma en estudio está compuesta de la siguiente forma:

Tabla 1. Composición de la estructura de capital de la empresa

\begin{tabular}{|l|c|c|c|c|c|c|c|c|c|c|}
\hline & $\begin{array}{c}\mathbf{2 0 0 7} \\
\text { Millones } \\
\text { de pesos }\end{array}$ & $\boldsymbol{\%}$ & $\begin{array}{c}\mathbf{2 0 0 8} \\
\text { Millones } \\
\text { de pesos }\end{array}$ & $\boldsymbol{\%}$ & $\begin{array}{c}\mathbf{2 0 0 9} \\
\text { Millones } \\
\text { de pesos }\end{array}$ & $\begin{array}{c}\mathbf{2 0 1 0} \\
\text { Millones } \\
\text { de pesos }\end{array}$ & $\begin{array}{c}\mathbf{2 0 1 1} \\
\text { Millones } \\
\text { de pesos }\end{array}$ & \% \\
\hline $\begin{array}{l}\text { Deuda a largo } \\
\text { largo plazo }\end{array}$ & 5225.8 & 37.1 & 4920.53 & 30 & 5883.19 & 32.7 & 5928.95 & 30.2 & 5930.18 & 28.1 \\
\hline Patrimonio & 8842.65 & 62.9 & 11438.15 & 70 & 12132.5 & 67.3 & 13679.41 & 69.8 & 15120.03 & 71.9 \\
\hline TOTAL & 14068.45 & 100 & 16358.68 & 100 & 18015.69 & 100 & 19608.36 & 100 & 21050.21 & 100 \\
\hline
\end{tabular}

Fuente: elaboración de los autores con base en los estados financieros de la firma en estudio. 
Se observa que en los cinco períodos estudiados la estructura de capital se mantuvo con una deuda cercana al $30 \%$, lo que muestra que la empresa es conservadora en mantener un nivel de deuda que no tiene cambios significativos en el tiempo.

\section{Costo de la deuda y del patrimonio}

El costo de la deuda representa el rendimiento que se debe pagar a los acreedores a cambio de los diferentes préstamos. Para hallar el costo de la deuda se utiliza un modelo matemático que calcula el valor a partir de datos extraídos de los estados financieros de la firma (ver Tabla 2).

$$
\mathrm{C}_{\mathrm{D}}=(\text { Intereses/Deuda }) *(1-\mathrm{T})
$$

En donde:

T representa la tasa del impuesto sobre la renta de las sociedades para el período comprendido entre los años 2007 a 2011 que en Colombia es del 34\% para el 2007, $33 \%$ a partir del año 2008 (DIAN, 2007).

Tabla 2. Costo de la deuda para los años 2007 a 2011

\begin{tabular}{|l|c|c|c|c|c|}
\hline & $\mathbf{2 0 0 7}$ & $\mathbf{2 0 0 8}$ & $\mathbf{2 0 0 9}$ & $\mathbf{2 0 1 0}$ & $\mathbf{2 0 1 1}$ \\
\hline Intereses & 123.67 & 164.43 & 281.79 & 316.21 & 325.94 \\
\hline Deuda & 5225.80 & 4920.53 & 5883.19 & 5928.95 & 5930.18 \\
\hline Impuesto de renta & 0.34 & 0.33 & 0.33 & 0.33 & 0.33 \\
\hline Costo de la deuda & $1.56 \%$ & $2.23 \%$ & $3.2 \%$ & $3.57 \%$ & $3.68 \%$ \\
\hline
\end{tabular}

Fuente: elaboración de los autores con base en los estados financieros y las notas a los estados financieros de la firma en estudio.

\section{Costo del patrimonio}

Según García (2003, p. 248), "el costo del patrimonio toma como referencia las tasas de interés del mercado, la rentabilidad de las empresas del sector y el riesgo país", su determinación constituye el aspecto de mayor complejidad en el cálculo del costo de capital. Para efectos del presente estudio se escoge realizar los cálculos del costo del patrimonio por el modelo CAPM ajustado, ya que es un método de uso muy popular entre los analistas y se tiene acceso a la información requerida. Según
García (2003) y Cruz et al., (2003) hallar el costo del patrimonio por el método CAPM resulta más complicado en economías pequeñas pues no se encuentra acceso a muchos datos y los betas no serían confiables, por tanto se toma como referencia mercados desarrollados y luego se realiza un ajuste con un riesgo adicional por operar en Colombia y no en dicho país $\left(\mathrm{R}_{\mathrm{P}}\right)$, como el resultado se obtiene en la moneda del país referencia, éste debe convertirse a pesos colombianos, a partir del uso de dos posibles métodos: aplicar la devaluación esperada a largo plazo o 
convirtiendo la obtenida en dólares a moneda constante y luego se aplica la inflación esperada a largo plazo. Esta alternativa es aceptada para valorar empresas en todo el mundo, tomando como referencia a Estados Unidos, por tener mercados bursátiles más grandes y eficientes. Para el presente estudio es necesario realizar los siguientes ajustes:

$$
\mathrm{K}_{\mathrm{e}}=\mathrm{R}_{\mathrm{f}}+\beta *\left(\mathrm{R}_{\mathrm{m}}-\mathrm{R}_{\mathrm{f}}\right)+\mathrm{R}_{\mathrm{P}}
$$

En donde:

$\beta$ : Representa el coeficiente de riesgo que asocia la volatilidad del rendimiento de la acción con la volatilidad de la rentabilidad del mercado (García, 2003). En el presente estudio se empezó de una beta desapalancada $\left(\beta_{\mathrm{U}}\right)$ que corresponde al valor del sector al que pertenece la empresa (Estados Unidos). Su valor fue hallado dentro de los cálculos presentados por Damodaran. Luego se calculan los valores correspondientes a las betas apalancadas, sus correspondientes resultados se hallan con la siguiente expresión:

$$
\beta=\beta_{\mathrm{U}}^{*}(1+(1-\mathrm{T}) *(\mathrm{D} / \mathrm{E}))
$$

En donde

T: representa la tasa del impuesto sobre la renta de las sociedades para el período comprendido entre los años 2007 a 2011 que en Colombia es del 34\% para el 2007 y del $33 \%$ para los siguientes años.
D/E: es el apalancamiento financiero o Leverage

$\mathrm{R}_{\mathrm{f}}$ : Según García (2003, p 262), "Es la tasa libre de riesgo asumida como la rentabilidad de los bonos del tesoro de los Estados Unidos con vencimiento en el largo plazo", generalmente se utilizan los de 30 años aunque hay quienes prefieren los de 10 y 20 años. En el presente estudio estos valores se toman como el rendimiento de los bonos del tesoro de Estados Unidos a largo plazo (10 años) tomados a diciembre 31 de los tres períodos estudiados.

$\mathrm{R}_{\mathrm{m}}-\mathrm{R}_{\mathrm{f}}$ : El $\mathrm{R}_{\mathrm{m}}$ representa el premio por el riesgo del mercado, es la tasa de rendimiento adicional que un inversionista obtiene por invertir en acciones en lugar de bonos del tesoro. Para García (2003) existe mucha controversia por parte de los académicos en el cálculo de este índice para encontrar el costo de capital, pues no hay acuerdo en el horizonte de tiempo que debe utilizarse para hallar dichos promedios. Los valores de $\mathrm{R}_{\mathrm{m}}-\mathrm{R}_{\mathrm{f}}$ para el presente estudio se calculan a partir de la diferencia entre el promedio histórico de los rendimientos obtenidos por invertir en acciones y el promedio histórico de la tasa libre de riesgo, desde el año 1928 hasta el año de análisis. Los datos son tomados de Damodaran Online (2010).

$\mathrm{R}_{\mathrm{p}}$ : representa el valor de riesgo del país, en este caso de Colombia. 
Teoría del Pecking O rder versusteoría del Trade off para la empresa Coservicios Sandra M ilena Zambrano V argas - Gustavo A dolfo A cuña Corredor

Finalmente,el resultado que se obtiene del $\mathrm{K}_{\mathrm{e}}$ se lleva a pesos colombianos usando la ecuación de Fisher, como lo explica García (2003, p. 271) así:

$\mathrm{K}_{\mathrm{e}(\text { Colombia })}=\left(1+\mathrm{K}_{\mathrm{e}(\mathrm{EU})}\right)(1+$ Devaluación $)-1$
Para hallar la devaluación se tiene que:

Devaluación $=(1+$ Inflación de Colombia $) /$ $(1+$ Inflación E. Unidos $)-1$

Los resultados obtenidos en el costo del patrimonio para Colombia por el método CAPM fueron los siguientes:

Tabla 3. Cálculo del patrimonio para Estados Unidos

\begin{tabular}{|c|c|c|c|c|c|c|c|c|c|c|}
\hline Año & $\mathbf{D}$ & $\mathbf{E}$ & $\beta_{\mathbf{U}}$ & $\mathbf{T}$ & $\mathbf{B}$ & $\mathbf{R}_{\mathrm{f}}$ & $\mathbf{R}_{\mathbf{m}}-\mathbf{R}_{\mathbf{f}}$ & $\mathbf{R}_{\mathbf{p}}$ & $\mathbf{K}_{\mathrm{e}(\mathrm{EU})}$ & $\mathbf{K}_{\mathrm{e}(\mathrm{Col})}$ \\
\hline $\mathbf{2 0 0 7}$ & 5225.8 & 8842.65 & 0.78 & 0.34 & 1.08 & 4.02 & 6.43 & 2.03 & 14.95 & 15.80 \\
$\mathbf{2 0 0 8}$ & 4920.53 & 11438.15 & 0.86 & 0.33 & 1.10 & 2.21 & 5.64 & 2.03 & 10.79 & 11.69 \\
$\mathbf{2 0 0 9}$ & 5883.19 & 12132.5 & 0.81 & 0.33 & 1.07 & 3.84 & 6.03 & 3.9 & 13.80 & 14.06 \\
$\mathbf{2 0 1 0}$ & 5928.95 & 13679.41 & 0.72 & 0.33 & 0.92 & 3.29 & 6.04 & 3.0 & 11.84 & 13.06 \\
$\mathbf{2 0 1 1}$ & 5930.18 & 15120.03 & 0.66 & 0.33 & 0.83 & 1.88 & 5.80 & 3.0 & 9.69 & 11.24 \\
\hline
\end{tabular}

Fuente: elaboración de los autores con base en Damodaran (2010) y Banco de la República (2011).

Para la empresa se observa que el valor más alto estuvo en el año 2007 y luego se disminuyó para el año 2008 y volvió a crecer para el 2009, esto puede explicarse en parte por la crisis financiera del 2008 que hizo que se redujera el consumo de nuestros productos en el exterior, que se redujera el consumo interno y que se redujeran los flujos de inversión extranjera (Banco de la República, 2010), que en suma causan una disminución en los retornos que deben pagarse a los propietarios de la firma.

\section{Cálculo del costo promedio ponde- rado de capital}

Para hallar el costo de capital se usa la metodología del costo promedio ponderado de capital que empieza con la determinación del costo de la deuda y el costo del capital contable. Después se halla la composición porcentual de cada uno de éstos elementos en el capital total de la empresa. Finalmente se halla el CPPC como la sumatoria de los productos de los pesos de la estructura de capital por su costo.

$$
\begin{aligned}
& \mathrm{CPPC}=\mathrm{C}_{\mathrm{D}} *(\mathrm{D} / \mathrm{V})+\mathrm{C}_{\mathrm{E}} *(\mathrm{E} / \mathrm{V}) \\
& \mathrm{V}=\mathrm{D}+\mathrm{E}
\end{aligned}
$$

En donde:

D: Representa el monto de la deuda E: Representa el monto de capital propio

Se calcula el costo de capital para los años 2007, 2008, 2009, 2010 y 2011 utilizando la metodología del CPPC. 
Tabla 4. CPPC para los años 2007 a 2011

\begin{tabular}{|c|c|c|c|c|}
\hline Año & Variable & Deuda & Patrimonio & Total \\
\hline \multirow[t]{3}{*}{2007} & $\begin{array}{l}\text { Composición de la estructura } \\
\text { de capital }\end{array}$ & $37,10 \%$ & $62,90 \%$ & \\
\hline & \begin{tabular}{|l|} 
Costo específico \\
\end{tabular} & $1,56 \%$ & $15,80 \%$ & \\
\hline & CPPC & $0,58 \%$ & $9,94 \%$ & $10,52 \%$ \\
\hline \multirow[t]{3}{*}{2008} & $\begin{array}{l}\text { Composición de la estructura } \\
\text { de capital }\end{array}$ & $30 \%$ & $70 \%$ & \\
\hline & Costo específico & $2,23 \%$ & $11,69 \%$ & \\
\hline & CPPC & $0,67 \%$ & $8,2 \%$ & $\mathbf{8 , 8 6 \%}$ \\
\hline \multirow[t]{3}{*}{2009} & $\begin{array}{l}\text { Composición de la estructura } \\
\text { de capital }\end{array}$ & $32,7 \%$ & $67,3 \%$ & \\
\hline & Costo específico & $3,2 \%$ & $14,06 \%$ & \\
\hline & CPPC & $1,05 \%$ & $9,47 \%$ & $10,52 \%$ \\
\hline \multirow[t]{3}{*}{2010} & \begin{tabular}{|l|} 
Composición de la estructura \\
de capital
\end{tabular} & $30,2 \%$ & $69,8 \%$ & \\
\hline & Costo específico & $3,57 \%$ & $13,06 \%$ & \\
\hline & CPPC & $1,07 \%$ & $9,11 \%$ & $10,18 \%$ \\
\hline 2011 & $\begin{array}{l}\text { Composición de la estructura } \\
\text { de capital } \\
\text { Costo específico } \\
\text { CPPC }\end{array}$ & $\begin{array}{l}28,1 \% \\
3,68 \% \\
1,03 \%\end{array}$ & $\begin{array}{c}71,9 \% \\
11,24 \% \\
8,08 \%\end{array}$ & $9,11 \%$ \\
\hline
\end{tabular}

Fuente: elaboración de los autores.

En el año 2008 se observa el menor costo de capital de los cinco períodos en estudio. Según García (2003), el patrimonio es la fuente más costosa y la deuda la más barata, en su conjunto generan un costo de capital inferior al costo del patrimonio. Se observa que en los cinco períodos estudiados la combinación de recursos fue más costosa que el patrimonio, en parte porque al ser el nivel de endeudamiento cercano al $30 \%$, dicha combinación no se beneficia de un valor de la deuda más bajo.

Análisis de la estructura de capital de acuerdo con las teorías propuestas

\section{Aplicación de la teoría del Trade Off}

Según la teoría del Trade Off, la estructura financiera de una firma está ligada a las ventajas fiscales de la deuda $\mathrm{y}$ al riesgo financiero que asume, de esta forma, a medida que se aumenta el nivel de la deuda se incrementan los beneficios impositivos, pero así mismo también se aumenta la obligación de pago de la deuda y las dificultades financieras (Miller, 1977). Cuando aumenta la deuda, los acreedores conscientes de la existencia de un mayor riesgo de impago exigen mayores tasas de interés y de igual forma los accionistas deben asumir 
Teoría del Pecking O rder versusteoría del Trade off para la empresa Coservicios Sandra M ilena Zambrano V argas - Gustavo A dolfo A cuña Corredor

mayor riesgo financiero y por tanto se incrementan sus expectativas. En su conjunto estas situaciones hacen que se incremente el costo de capital y que se disminuya el valor de la empresa en el mercado. Se puede concluir entonces, según la teoría del Trade Off, que debe existir un punto óptimo de endeudamiento en donde el costo de capital sea mínimo y así mismo el valor de la empresa sea máximo.

\section{Modelo propuesto por López y De Luna (2002)}

Según López y De Luna (2002) a través de MM y el CAPM puede obtenerse una solución aproximada para determinar el nivel de endeudamiento que minimice el CPPC, para ello se puede partir de la estructura actual de la firma y estimar la evolución del costo de la deuda de acuerdo con diversos grados de endeudamiento y hallando el costo de capital por el método financiero y el cálculo del patrimonio por el método de la Beta Apalancada (García, 2003).

Para el cálculo del CPPC para los años 2007 a 2011 en la empresa se tuvieron en cuenta las siguientes variables:

- D/(D+E): Muestra el nivel de endeudamiento de la firma, para el presente caso se tienen en cuenta los niveles de endeudamiento del $10 \%$, $20 \%, 30 \%, 40 \%, 50 \%$ y $60 \%$.

- E: Representa los valores correspondientes al 100\%, 90\%, 80\%,
$70 \%, 60 \%, 50 \%$ y $40 \%$ de la sumatoria de deuda a largo plazo y patrimonio mostrados en la Tabla 1.

- D: Representa los valores correspondientes al $0 \%, 10 \%, 20 \%$, $30 \%, 40 \%, 50 \%$ y $60 \%$ de la sumatoria de deuda a largo plazo y patrimonio mostrados en la Tabla 1.

- $\quad \beta_{\mathrm{U}}$ : Representa la beta desapalancada para el sector Water Utility (en Estados Unidos). Su valor fue hallado dentro de los cálculos presentados por Damodaran.

- D/E: Representa la proporción de deuda sobre patrimonio.

- $\quad \beta$ : Representa el coeficiente de riesgo asociado con cada uno de los niveles de endeudamiento. Se calcula de la siguiente manera: $\beta=\beta_{\mathrm{U}}^{*}(1+(1-\mathrm{T}))$. En donde $\mathrm{T}$ representa la tasa del impuesto sobre la renta de las sociedades para el período comprendido entre los años 2007 a 2011.

- $R_{\mathrm{f}}, \mathrm{R}_{\mathrm{m}}-\mathrm{R}_{\mathrm{f}} \mathrm{y} \mathrm{R}_{\mathrm{p}}$ toman sus valores de la Tabla 3.

- Para hallar los valores del $\mathrm{K}_{\mathrm{e}(\mathrm{Col})}$ y el $\mathrm{K}_{\mathrm{e}(\mathrm{EU})}$ se utiliza el mismo procedimiento que el mostrado para encontrar estos datos en la tabla 3.

- Cd: El costo de la deuda usado, se supone que aumenta a medida que también lo hace el nivel de endeudamiento ya que se considera que el grado de riesgo se incrementa y por tanto, se espera que los acreedores cobren mayores tasas de interés (Cruz, 2003, p.404). Para la realización de este trabajo se consideran las siguientes suposiciones: 
como valor de la deuda del $60 \%$ se toma el valor mayor alcanzado por la tasa de colocación del mismo año en las bases de datos del Banco de la República, los valores para los otros niveles de endeudamiento $(10 \%, 20 \%$, $30 \%, 40 \%$ y $50 \% 60 \%$ ) se toman proporcionalmente con otras tasas de interés de colocación. Las tasas de deuda escogidas y afectadas por el impuesto de renta para cada uno de los períodos analizados se muestran a continuación:

Tabla 5. Costo de la deuda supuesto para los años 2007 a 2011

\begin{tabular}{|c|c|c|c|c|c|c|c|c|c|c|}
\hline \multirow{2}{*}{$\begin{array}{c}\text { Nivel } \\
\text { de End. }\end{array}$} & \multicolumn{2}{|c|}{2007} & \multicolumn{2}{|c|}{2008} & \multicolumn{2}{|c|}{2009} & \multicolumn{2}{|c|}{2010} & \multicolumn{2}{|c|}{2011} \\
\cline { 2 - 10 } & Cd & Cd(1-T) & Cd & Cd(1-T) & Cd & Cd(1-T) & Cd & Cd(1-T) & Cd & Cd(1-T) \\
\hline $10 \%$ & $3,90 \%$ & $2,57 \%$ & $5,97 \%$ & $4,00 \%$ & $3,50 \%$ & $2,35 \%$ & $4,23 \%$ & $2,83 \%$ & $5,22 \%$ & $3,49 \%$ \\
$20 \%$ & $5,80 \%$ & $3,83 \%$ & $7,20 \%$ & $4,82 \%$ & $7,50 \%$ & $5,03 \%$ & $6,72 \%$ & $4,50 \%$ & $7,49 \%$ & $5,01 \%$ \\
$30 \%$ & $8,80 \%$ & $5,81 \%$ & $8,80 \%$ & $5,90 \%$ & $8,00 \%$ & $5,36 \%$ & $8,33 \%$ & $5,58 \%$ & $9,26 \%$ & $6,20 \%$ \\
$40 \%$ & $9,90 \%$ & $6,53 \%$ & $9,50 \%$ & $6,37 \%$ & $10,00 \%$ & $6,70 \%$ & $9,58 \%$ & $6,41 \%$ & $11,02 \%$ & $7,38 \%$ \\
$50 \%$ & $12,50 \%$ & $8,25 \%$ & $12,50 \%$ & $8,38 \%$ & $11,60 \%$ & $7,77 \%$ & $10,46 \%$ & $7,00 \%$ & $12,33 \%$ & $8,26 \%$ \\
$60 \%$ & $14,31 \%$ & $9,44 \%$ & $16,36 \%$ & $10,96 \%$ & $12,40 \%$ & $8,31 \%$ & $13,44 \%$ & $9,00 \%$ & $15,73 \%$ & $10,5 \%$ \\
\hline
\end{tabular}

Fuente: elaboración de los autores a partir de Base de Datos Banco de la República

- Finalmente, el CPPC se halla utilizando la siguiente fórmula:

$\mathrm{CPPC}=\mathrm{C}_{\mathrm{D}} *(\mathrm{D} / \mathrm{V})+\mathrm{K}_{\mathrm{e}(\mathrm{Col})} *(\mathrm{E} / \mathrm{V})$

En donde:
D: Representa el monto de la deuda E: Representa el monto de capital propio

A continuación se muestran los resultados obtenidos luego de aplicar los anteriores cálculos: 
Teoría del Pecking O rder versusteoría del Trade off para la empresa Coservicios Sandra M ilena Zambrano V argas - Gustavo A dolfo A cuña Corredor

Tabla 6. Cálculo del CPPC en la empresa de acuerdo con diferentes niveles de deuda

\begin{tabular}{|c|c|c|c|c|c|c|c|c|c|c|c|c|c|}
\hline AÑO & $\begin{array}{c}\text { D/ } \\
(\mathbf{D}+\mathbf{E})\end{array}$ & D & $\mathbf{E}$ & $\beta_{\mathbf{U}}$ & $\mathrm{D} / \mathrm{E}$ & $\beta$ & $\mathbf{R}_{\mathrm{f}}$ & $\mathbf{R}_{\mathrm{m}}-\mathbf{R}_{\mathrm{f}}$ & $\mathbf{R}_{p}$ & $\mathbf{K}_{\mathrm{e}(\mathbf{E U})}$ & $\mathbf{K}_{\mathrm{e}(\mathrm{COL})}$ & Cd*(1-T & СРРC \\
\hline \multirow[t]{7}{*}{2007} & $0 \%$ & 0 & 14068,45 & \multirow[t]{7}{*}{0.78} & 0 & 0,78 & 4,02 & \multirow[t]{7}{*}{6,49} & \multirow[t]{7}{*}{3,9} & 12,9 & 13,7 & 0,00 & 0,1373 \\
\hline & $10 \%$ & 1406,85 & 12661,61 & & 0,111 & 0,84 & & & & 13,3 & 14,1 & 0,02 & 0,1297 \\
\hline & $20 \%$ & 2813,69 & 11254,76 & & 0,250 & 0,91 & & & & 13,8 & 14,6 & 0,03 & 0,1246 \\
\hline & $30 \%$ & 4220,54 & 9847,92 & & 0,429 & 1,00 & & & & 14,4 & 15,2 & 0,05 & 0,1242 \\
\hline & $40 \%$ & 5627,38 & 8441,07 & & 0,667 & 1,13 & & & & 15,2 & 16,1 & 0,06 & 0,1228 \\
\hline & $50 \%$ & 7034,23 & 7034,23 & & 1,000 & 1,30 & & & & 16,3 & 17,3 & 0,08 & 0,1278 \\
\hline & $60 \%$ & 8441,07 & 5627,38 & & 1,500 & 1,56 & & & & 18,0 & 19,0 & 0,09 & 0,1330 \\
\hline \multirow[t]{7}{*}{2008} & $0 \%$ & 0 & 16358,68 & \multirow[t]{7}{*}{0.86} & 0 & 0,86 & 2,21 & \multirow[t]{7}{*}{5,92} & \multirow[t]{7}{*}{2,03} & 9,33 & 10,1 & 0,00 & 0,1012 \\
\hline & $10 \%$ & 1635,87 & 14722,81 & & 0,111 & 0,92 & & & & 9,71 & 10,5 & 0,04 & 0,0987 \\
\hline & $20 \%$ & 3271,74 & 13086,94 & & 0,250 & 1,00 & & & & 10,1 & 11,0 & 0,04 & 0,0979 \\
\hline & $30 \%$ & 4907,60 & 11451,08 & & 0,429 & 1,11 & & & & 10,7 & 11,6 & 0,05 & 0,0995 \\
\hline & $40 \%$ & 6543,47 & 9815,21 & & 0,667 & 1,24 & & & & 11,6 & 12,5 & 0,06 & 0,1008 \\
\hline & $50 \%$ & 8179,34 & 8179,34 & & 1,000 & 1,44 & & & & 12,7 & 13,7 & 0,08 & 0,1108 \\
\hline & $60 \%$ & 9815,21 & 6543,47 & & 1,500 & 1,72 & & & & 14,4 & 15,6 & 0,11 & 0,1283 \\
\hline \multirow[t]{7}{*}{2009} & $0 \%$ & 0 & 18015,69 & \multirow[t]{7}{*}{0.81} & 0 & 0,81 & 3,84 & \multirow[t]{7}{*}{6,12} & \multirow[t]{7}{*}{3,4} & 12,2 & 12,4 & 0,00 & 0,1243 \\
\hline & $10 \%$ & 1801,57 & 16214,12 & & 0,111 & 0,87 & & & & 12,5 & 12,8 & 0,02 & 0,1176 \\
\hline & $20 \%$ & 3603,14 & 14412,55 & & 0,250 & 0,95 & & & & 13,0 & 13,2 & 0,05 & 0,1163 \\
\hline & $30 \%$ & 5404,71 & 12610,98 & & 0,429 & 1,04 & & & & 13,6 & 13,8 & 0,05 & 0,1132 \\
\hline & $40 \%$ & 7206,28 & 10809,41 & & 0,667 & 1,17 & & & & 14,4 & 14,6 & 0,06 & 0,1149 \\
\hline & $50 \%$ & 9007,85 & 9007,85 & & 1,000 & 1,35 & & & & 15,5 & 15,8 & 0,07 & 0,1179 \\
\hline & $60 \%$ & 10809,4 & 7206,28 & & 1,500 & 1,62 & & & & 17,1 & 17,5 & 0,08 & 0,1199 \\
\hline \multirow[t]{7}{*}{2010} & $0 \%$ & 0 & 19608,30 & \multirow[t]{7}{*}{0.72} & 0 & 0,72 & 3.29 & \multirow[t]{7}{*}{6.04} & \multirow[t]{7}{*}{3.0} & 10,6 & 11,1 & 0,03 & 0,1119 \\
\hline & $10 \%$ & 1960,83 & 17647,47 & & 0,111 & 0,77 & & & & 10,9 & 11,5 & 0,02 & 0,1066 \\
\hline & $20 \%$ & 3921,66 & 15686,64 & & 0,250 & 0,84 & & & & 11,3 & 11,9 & 0,04 & 0,1046 \\
\hline & $30 \%$ & 5882,49 & 13725,81 & & 0,429 & 0,93 & & & & 11,8 & 12,4 & 0,05 & 0,1042 \\
\hline & $40 \%$ & 7843,32 & 11764,98 & & 0,667 & 1,04 & & & & 12,5 & 13,2 & 0,06 & 0,1050 \\
\hline & $50 \%$ & 9804,15 & 9804,15 & & 1,000 & 1,20 & & & & 13,5 & 14,2 & 0,07 & 0,1062 \\
\hline & $60 \%$ & 11764,98 & 7843,32 & & 1,500 & 1,44 & & & & 15,0 & 15,7 & 0,09 & 0,1171 \\
\hline \multirow[t]{7}{*}{2011} & $0 \%$ & 0 & 21050,20 & \multirow[t]{7}{*}{0.66} & 0 & 0,66 & 1.88 & \multirow[t]{7}{*}{5.8} & \multirow[t]{7}{*}{3.0} & 8,71 & 9,36 & 0 & 0,0936 \\
\hline & $10 \%$ & 2105,02 & 18945,18 & & 0,111 & 0,71 & & & & 8,99 & 9,66 & 0,03 & 0,0920 \\
\hline & $20 \%$ & 4210,04 & 16840,16 & & 0,250 & 0,77 & & & & 9,35 & 10,0 & 0,05 & 0,0927 \\
\hline & $30 \%$ & 6315,06 & 14735,14 & & 0,429 & 0,85 & & & & 9,81 & 10,5 & 0,06 & 0,0959 \\
\hline & $40 \%$ & 8420,08 & 12630,12 & & 0,667 & 0,95 & & & & 10,4 & 11,1 & 0,07 & 0,1001 \\
\hline & $50 \%$ & 10525,1 & 10525,10 & & 1,000 & 1,10 & & & & 11,2 & 12,1 & 0,08 & 0,1130 \\
\hline & $60 \%$ & 12630,12 & 8420,08 & & 1,500 & 1,32 & & & & 12,5 & 13,4 & 0,10 & 0,0539 \\
\hline
\end{tabular}

Fuente: elaboración de los autores con base en los estados financieros de la empresa y de Damodaran (2010).

\section{Conclusiones del presente modelo}

Para el año 2007, el costo de capital más bajo se presentó en un nivel de endeudamiento del $40 \%$, en el 2008 fue del $20 \%$ y en el 2009 del $30 \%$. Si observamos el nivel de endeudamiento real de la firma mostrado en la estructura de capital (ver Tabla 1) para los mismos años, estos fueron respectivamente del $37,1 \%, 30 \%$ y $32,7 \%, 30,2 \%$ y $28,1 \%$.

En el 2007, 2009 y 2010, los valores óptimos y reales de endeudamiento estuvieron muy cercanos, mientras que en el 2008 tuvieron una diferencia de diez 
puntos porcentuales y en el 2011 una diferencia del $18 \%$, indicando que para dichos períodos la firma no cumplió el objetivo de estructura óptima.

El modelo estudiado posee una gran deficiencia que hace que en parte pierda validez, pues supone que el flujo de caja libre es independiente de la deuda debido a que explica que el apalancamiento operativo es independiente del nivel de deuda que se posea. Eso en parte es cierto cuando hay variaciones marginales en los niveles de endeudamiento razonables de la estructura de capital, sin embargo, cuando hay grandes variaciones en ese endeudamiento, el FCL se verá afectado seriamente. Esto se debe entre otros motivos a que aparecen costos adicionales relacionados con la quiebra, costos de agencia por los conflictos entre los diferentes actores y que se pierda eficacia en la gestión directiva (López \& De Luna, 2002).

Finalmente, el hecho de generar un nivel de endeudamiento en donde el costo de capital sea el más bajo, no garantiza que la empresa pueda cubrir el servicio de la deuda a ese nivel.

\section{Modelo propuesto por Cruz y otros} (2003)

Para estudiar la estructura financiera de una empresa se tienen en cuenta cinco escenarios posibles de la economía con una tendencia de probabilidad normal, los cuales se establecen de acuerdo con la rentabilidad del mercado. Dichos escenarios en el presente estudio se van a denominar como: malo, regular, aceptable, bueno y excelente (Cruz, 2003). Las probabilidades de ocurrencia de cada uno de estos escenarios se determinaron de acuerdo con condiciones de oferta y demanda de los servicios prestados por la firma y de proyecciones de ventas futuras, como se explica a continuación:

La empresa en estudio es un monopolio natural debido a la clase de servicios que presta, por tanto se determina como demanda aceptable una probabilidad del $50 \%$, ya que se supone que los cambios en cuanto al incremento de suscriptores crece en volúmenes pequeños lo que no le significa incrementos con alta variabilidad en los ingresos de la firma. Para las demandas regular y buena se va a establecer una probabilidad del $20 \%$ respectivamente, que se supone sucede si se disminuyen o incrementan los ingresos por venta de servicios en un $10 \%$. Por último, las demandas mala y excelente en el presente trabajo tienen un valor del $5 \%$ cada una, cantidad que representa la posibilidad de que el número de suscriptores crezca de manera extraordinaria si hubiese en la ciudad un incremento sustancial de las construcciones que conllevaría al aumento de matrículas de acueducto, o por el contrario, a una disminución exagerada de suscriptores que no consumieran el servicio lo que para la ciudad sería poco probable. 
Teoría del Pecking O rder versusteoría del Trade off para la empresa Coservicios Sandra M ilena Zambrano V argas - Gustavo A dolfo A cuña Corredor

Cálculo de las utilidades operacionales años 2007, 2008, 2009, 2010 y 2011

Para hallar las utilidades operacionales de cada uno de los años en estudio, se realizó una simulación con los posibles escenarios,para ello se tienen en cuenta las utilidades operacionales obtenidas en los estados financieros durante los tres períodos para la demanda aceptable, debido a que el valor obtenido en dichas utilidades es el más probable que ocurra porque se tienen unos cálculos de ingresos proyectados de acuerdo con las tarifas y el consumo promedio de los diferentes servicios y de acuerdo con la eficiencia operativa de la compañía. Estas utilidades nos sirven de base para encontrar las utilidades en los otros escenarios. Para las demandas regular y buena se establece una disminución y un incremento del $5 \%$ de la demanda aceptable y finalmente para las demandas mala y excelente una disminución y un incremento del $10 \%$ con respecto a la misma demanda. De esta forma se tendría:

- La utilidad para la demanda aceptable es la correspondiente a la utilidad operacional para los años 2007, 2008, 2009, 2010 y 2011 en el estado de resultados.

- La utilidad para la demanda mala es igual a demanda aceptable* $(1-0,10)$

- La utilidad para la demanda regular es igual a demanda aceptable*(1-0,05)

- La utilidad para la demanda buena es igual a demanda aceptable* $(1+0,05)$

- La utilidad para la demanda excelente es igual a demanda aceptable $*(1+0,10)$

A continuación se muestran los resultados obtenidos:

Tabla 7. Cálculo de la utilidad operacional según escenarios de demanda (en millones de pesos)

\begin{tabular}{|l|c|c|c|c|c|}
\hline DEMANDA & $\mathbf{2 0 0 7}$ & $\mathbf{2 0 0 8}$ & $\mathbf{2 0 0 9}$ & $\mathbf{2 0 1 0}$ & $\mathbf{2 0 1 1}$ \\
\hline Mala & 1073.43 & 887.17 & 1225.95 & 1300,08 & 450,42 \\
Regular & 1133.07 & 936.46 & 1294.06 & 1372,31 & 475,45 \\
Aceptable & 1192.70 & 985.75 & 1362.17 & 1444,54 & 500,47 \\
Buena & 1252.34 & 1035.04 & 1430.28 & 1516,76 & 525,49 \\
Excelente & 1311.97 & 1084.32 & 1498.39 & 1588,99 & 550,52 \\
\hline
\end{tabular}

Fuente: elaboración de los autores con base en los estados financieros de la empresa.

Con base en los datos hallados en la tabla anterior para los períodos 2007, 2008, 2009,2010 y 2011 y de acuerdo con los diferentes escenarios de la economía, se procede a calcular las UPA para cada nivel de endeudamiento. El procedimiento utilizado es el siguiente:

- Se parte de las utilidades correspondientes a la Tabla 7como utilidades operacionales (UAII). 
- Se buscan los intereses de la deuda aplicando el valor correspondiente al costo de la deuda explicado en la Tabla 5 (ya afectados por la tasa de impuesto de renta) y se le aplican al valor de la deuda simulada que corresponde al nivel de endeudamiento analizado $(10 \%, 20 \%, 30 \%$, $40 \%, 50 \%$ y $60 \%$ ) sobre el total de los activos para ese año.

- Luego se halla la utilidad antes de impuestos que corresponde a la utilidad operacional menos los intereses.

- La utilidad neta se encuentra al afectar la anterior utilidad con el impuesto de renta.

- Finalmente se halla la utilidad por acción dividiendo el total de la utilidad neta entre el número de acciones de la empresa que para los cinco años en estudio fueron de 941.857 .
- Al realizar estos cálculos de acuerdo con un nivel de deuda se busca la esperanza matemática de las UPA hallando la sumatoria de la multiplicación de cada UPA por su probabilidad de ocurrencia (de acuerdo con cada escenario de la economía). Esa esperanza matemática se constituye en el valor de la UPA por cada nivel de endeudamiento en el respectivo período y que nos servirá de base para hacer la comparación entre el valor mayor de la UPA y el valor menor del CPPC en un determinado nivel de deuda.

El objetivo del modelo explicado por Cruz et al., (2003) se condensa en la siguiente tabla (años 2007, 2008, 2009, 2010 y 2011), en la que se busca analizar si se cumplió la teoría del Trade Off, en donde el valor del CPPC debe ser mínimo en el mismo nivel de endeudamiento y en donde el valor de la UPA sea máximo. Los resultados junto con sus conclusiones se muestran a continuación: 
Teoría del Pecking O rder versusteoría del Trade off para la empresa Coservicios Sandra M ilena Zambrano V argas - Gustavo A dolfo A cuña Corredor

Tabla 8. Resumen estructura de capital óptima años 2007, 2008, 2009, 2010 y 2011

\begin{tabular}{|c|c|c|c|c|c|}
\hline Año & $\begin{array}{c}\text { Nivel de } \\
\text { Endeudamiento }\end{array}$ & $\begin{array}{l}\text { (UPA) } \\
\text { Pesos }\end{array}$ & $\mathbf{K}_{\mathrm{e}}(\%)$ & $\begin{array}{r}\mathbf{U P A}_{\mathbf{K}} \\
\text { (pesos) }\end{array}$ & $\begin{array}{c}\text { CPPC } \\
(\%)\end{array}$ \\
\hline 2007 & $\begin{array}{c}0 \% \\
10 \% \\
20 \% \\
30 \% \\
40 \% \\
50 \% \\
60 \% \\
\end{array}$ & $\begin{array}{c}835,78 \\
861,59 \\
789,1 \\
506,02 \\
178,5 \\
-801,6 \\
-1897,29\end{array}$ & $\begin{array}{c}13,73 \\
14,13 \\
14,62 \\
15,26 \\
16,11 \\
17,3 \\
19,09\end{array}$ & $\begin{array}{c}60,88 \\
61 \\
53,97 \\
33,16 \\
11,08 \\
-46,33 \\
-99,4\end{array}$ & $\begin{array}{c}0,1373 \\
0,1297 \\
0,1246 \\
0,1242 \\
0,1228 \\
0,1278 \\
0,133\end{array}$ \\
\hline 2008 & $\begin{array}{c}0 \% \\
10 \% \\
20 \% \\
30 \% \\
40 \% \\
50 \% \\
60 \%\end{array}$ & $\begin{array}{c}701,22 \\
637,16 \\
486,7 \\
184,96 \\
-202,92 \\
-1626,03 \\
-3561,64\end{array}$ & $\begin{array}{l}10,12 \\
10,52 \\
11,03 \\
11,69 \\
12,56 \\
13,79 \\
15,62\end{array}$ & $\begin{array}{c}75,15 \\
65,62 \\
47,79 \\
17,14 \\
-17,49 \\
-127,61 \\
-246,52\end{array}$ & $\begin{array}{l}0,1012 \\
0,0987 \\
0,0979 \\
0,0995 \\
0,1008 \\
0,1108 \\
0,1283\end{array}$ \\
\hline 2009 & $\begin{array}{c}0 \% \\
10 \% \\
20 \% \\
30 \% \\
40 \% \\
50 \% \\
60 \% \\
\end{array}$ & $\begin{array}{c}968,99 \\
966,7 \\
681,08 \\
414,84 \\
-270,03 \\
-1672,43 \\
-2836,72 \\
\end{array}$ & $\begin{array}{c}12,43 \\
12,81 \\
13,28 \\
13,88 \\
14,68 \\
15,81 \\
17,5 \\
\end{array}$ & $\begin{array}{c}79,44 \\
76,93 \\
52,28 \\
30,46 \\
-18,74 \\
-107,77 \\
-165,13 \\
\end{array}$ & $\begin{array}{l}0,1243 \\
0,1176 \\
0,1163 \\
0,1132 \\
0,1149 \\
0,1179 \\
0,1199 \\
\end{array}$ \\
\hline 2010 & $\begin{array}{c}0 \% \\
10 \% \\
20 \% \\
30 \% \\
40 \% \\
50 \% \\
60 \% \\
\end{array}$ & $\begin{array}{c}1027,59 \\
1026,40 \\
797,53 \\
581,56 \\
-134,70 \\
-845,11 \\
-1567,34\end{array}$ & $\begin{array}{l}11,19 \\
11,53 \\
11,95 \\
12,49 \\
13,22 \\
14,24 \\
15,76\end{array}$ & $\begin{array}{c}91,86 \\
89,01 \\
66,73 \\
46,56 \\
-10,18 \\
-59,34 \\
-99,45\end{array}$ & $\begin{array}{l}0,1119 \\
0,1066 \\
0,1046 \\
0,1042 \\
0,1050 \\
0,1062 \\
0,1171\end{array}$ \\
\hline 2011 & $\begin{array}{c}0 \% \\
10 \% \\
20 \% \\
30 \% \\
40 \% \\
50 \% \\
60 \%\end{array}$ & $\begin{array}{c}356,01 \\
326,29 \\
140,58 \\
-215,66 \\
-345,71 \\
-887,94 \\
-1895,32\end{array}$ & $\begin{array}{c}9,36 \\
9,66 \\
10,04 \\
10,53 \\
11,18 \\
12,10 \\
13,46\end{array}$ & $\begin{array}{c}38,03 \\
33,77 \\
14,00 \\
-20,48 \\
-30,92 \\
-73,38 \\
-140,81\end{array}$ & $\begin{array}{l}0,0936 \\
0,0920 \\
0,0927 \\
0,0959 \\
0,1001 \\
0,1130 \\
0,0539\end{array}$ \\
\hline
\end{tabular}

Fuente: elaboración de los autores a partir de cálculos anteriores. 


\section{Conclusiones del presente modelo}

- Para el 2007 se muestra que la empresa no encontró un nivel óptimo de deuda, ya que su mayor valor de la UPA se encontró con el $10 \%$ de endeudamiento. De ahí en adelante, el valor de la UPA siempre bajó en la medida que aumentaba el nivel de endeudamiento. Como el valor más bajo de costo de capital se dio en el $40 \%$ de endeudamiento, se concluye que en este año la estructura de capital no se ajusta a la teoría del Trade Off, según la cual cuando la empresa adquiere su máximo valor en el mercado, así mismo tiene el menor costo de capital (Shyam\& Myers, 1994).

- Para el año 2008, se encontró un punto de deuda óptima ubicada en el $0 \%$ en donde el valor de la UPA es máximo, pero el costo de capital es mínimo en un nivel de endeudamiento del $20 \%$. Si comparamos este resultado con la estructura de capital de la firma para ese año, encontramos que la empresa tuvo un nivel de deuda del $30 \%$, es decir que no se ajusta al igual que en el 2007 a la teoría del Trade Off porque el nivel de endeudamiento ideal debería estar diez puntos por debajo de lo que en realidad estuvo.

- En el 2009, no se encontró un punto óptimo de deuda, pues mientras el valor de la empresa fue mayor en un nivel de deuda del $0 \%$, el valor más bajo de costo de capital se presentó en un nivel de deuda del 30\%. En conclusión y con los valores supuestos, la firma no cumplió con los parámetros de la teoría del Trade Off en dicho año.

- La implementación del presente modelo presenta debilidades debido a que se involucra un alto juicio de subjetividad en cuanto al establecimiento de la probabilidad de ocurrencia de cada uno de los escenarios de la economía y por tanto en el cálculo de la utilidad por acción. Según los planteamientos teóricos, un costo de capital mínimo debe conllevar a una utilidad por acción máxima, en la realidad observamos que para el caso de la empresa en estudio eso no sucede y se manifiesta una inconsistencia entre esas dos variables no pudiendo llegar a un nivel de deuda óptimo.

- Al utilizar la misma lógica en el cálculo del CPPC, el resultado es el mismo nivel de endeudamiento con los dos modelos. Sin embargo, al igual que con el modelo de López y De Luna (2002), no se combina ningún criterio que analice si la empresa está en condiciones de cubrir el servicio de la deuda acorde con el nivel de endeudamiento que se encontró como óptimo.

\section{Análisis de los resultados encontrados de acuerdo con la teoría del Trade Off}

- La teoría del equilibrio estático afirma que las empresas tienen un nivel de endeudamiento óptimo en donde se compensan las ventajas (deducciones 
impositivas) y las desventajas (costos de dificultades financieras y costos de agencia) de usar la deuda (Jensen \& Meckling, 1976). Se espera que la firma encuentre ese óptimo y poco a poco se acomode en ese nivel de deuda, que si en la práctica es bajo, pueda aumentarse para realizar nuevos proyectos de inversión, o si está muy alto, logre bajarse recurriendo a otras fuentes de financiación como los recursos internos.

- Es importante recalcar que aún no existe un modelo matemático que brinde las posibilidades para encontrar el nivel de deuda óptima que maximice el valor de la firma. Para que se cumpla la teoría del Trade Off, es necesario que el valor de la empresa sea máximo y el costo de capital mínimo. Desafortunadamente, en la práctica no siempre ocurre lo que la teoría plantea y resulta difícil acomodar un modelo a una realidad con su sinnúmero de probabilidades.

- En los dos modelo estudiados existe una deficiencia principal que es no tener en cuenta el FCL a la hora de determinar el nivel de endeudamiento óptimo, pues la modelación del problema nos puede arrojar un nivel de deuda que en la práctica la firma no estaría en capacidad de cubrir. Por ello es necesario buscar y aplicar condiciones que incluyan aspectos como: la capacidad de la empresa para generar flujo de caja libre, el plazo y la tasa de interés de la deuda, la política de dividendos, el objetivo de calificación y la capacidad de respaldo de la deuda como lo afirma García (2003), quien es más específico en aceptar las debilidades de algunas aplicaciones para el Trade Off.

- No se debe olvidar que la empresa, por ser regulada, depende de las tarifas que le imponga el ente regulador para conocer su nivel de ingresos, por tanto puede no tener incentivos para manejar sus niveles de deuda, y por otro lado, el hecho de ser mixta y en su mayoría propiedad del municipio de Sogamoso, hace que los directivos no necesariamente busquen lograr un máximo de utilidades económicas sino más bien sociales, garantizando servicios públicos de alta calidad y con precios relativamente competitivos para los usuarios del municipio.

- Para Frank y Goyal (2007), los estudios empíricos encuentran una relación negativa entre rentabilidad y apalancamiento. El Trade Off predice que las firmas más grandes deberían tener mayor deuda. En el caso estudiado, la rentabilidad disminuye en los años analizados, solamente se incrementa en el 2009 un poco, pero no permite llevar a concluir esta relación. Es probable que la rentabilidad de los activos tan baja sea el resultado de la falta de gestión sobre activos improductivos. Por otro lado, también se predice que existe una relación positiva entre el apalancamiento y el grado en que los activos se utilizan como garantía de la deuda. 


\section{Aplicación de la teoría del Pecking Order}

La teoría del Pecking Order se basa en las preferencias que tienen los directivos de las firmas en cuanto al uso de fuentes internas y externas de financiación para nuevos proyectos. Se asume que el nivel de endeudamiento está alterado por la cantidad de dichas fuentes, las internas con una relación inversa, es decir, a mayores fuentes internas, menor endeudamiento. En este aparte del estudio se busca demostrar que la empresa durante los años 2007 a 2011 siguió los planteamientos de la teoría del Pecking Order, esto es, cuando requirió fondos para nuevos proyectos de inversión, en primer lugar prefirió utilizar sus fondos internos, si no le fue suficiente recurrió a la deuda y por último a la emisión de acciones. Esta última opción no se estudia, ya que la empresa no ha emitido nuevas acciones desde su creación y a medio plazo no tiene pensado como estrategia de obtención de recursos la emisión de las mismas.

Para la aplicación de la teoría se van a tener en cuenta las siguientes variables:

- Como variable dependiente se propone el nivel de endeudamiento a largo plazo.

\section{Nivel de endeudamiento a largo plazo}

Este indicador busca analizar cuántos de los activos de operación de la firma son propiedad de los acreedores. Se asume como pasivos a largo plazo solo los financieros, porque son estos los que interesan en la estructura de capital, y como activos solo los de operación por estar relacionados con el propósito de la firma.

Los activos operacionales netos de la empresa para los cinco períodos en estudio,se muestran en la Tabla 9:

Tabla 9. Lista de activos operacionales netos

\begin{tabular}{|c|}
\hline ACTIVOSOPERACIONALES NETOS \\
\hline Efectivo \\
Deudores por servicios \\
Avances y anticipos \\
Inventarios \\
Gastos pagados por anticipado \\
Otros deudores \\
Deudores no corrientes \\
Propiedad planta y equipo \\
Activos adquiridos por leasing \\
Intangibles \\
\hline
\end{tabular}

Fuente: elaboración de los autores con base en los estados financieros de la empresa. 
El nivel de endeudamiento a largo plazo

Endeudamiento $=$ pasivos financieros $\mathrm{a}$ se calcula con la siguiente relación:

largo plazo/AON[9]

Tabla 10. Endeudamiento de la empresa en millones de pesos

\begin{tabular}{|l|c|c|c|c|c|}
\hline & $\mathbf{2 0 0 7}$ & $\mathbf{2 0 0 8}$ & $\mathbf{2 0 0 9}$ & $\mathbf{2 0 1 0}$ & $\mathbf{2 0 1 1}$ \\
\hline $\begin{array}{l}\text { Pasivos financieros a } \\
\text { largo plazo }\end{array}$ & 5225.80 & 4920.53 & 5883.19 & 5928.95 & 5930.18 \\
\hline AON & 24071.36 & 28172.46 & 37305.56 & 43414.73 & 37470.41 \\
\hline Endeudamiento & $\mathbf{2 1 . 7 \%}$ & $\mathbf{1 7 . 4 6 \%}$ & $\mathbf{1 5 . 7 7 \%}$ & $\mathbf{1 3 . 6 5 \%}$ & $\mathbf{1 5 . 8 2 \%}$ \\
\hline
\end{tabular}

Fuente: elaboración de los autores con base en los estados financieros de la empresa.

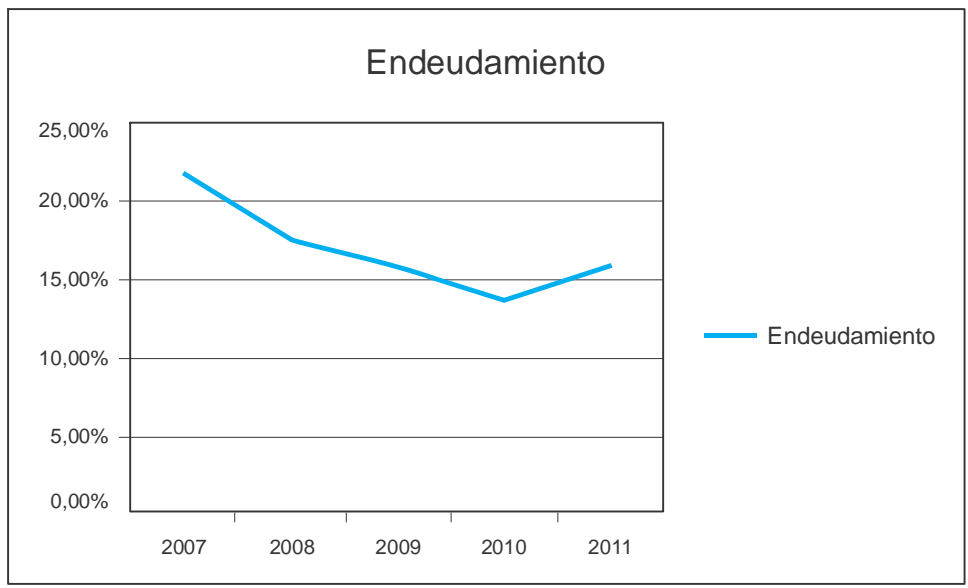

Figura 1. Endeudamiento.

Fuente: elaboración de los autores con base en los estados financieros de la empresa.

Se observa que el endeudamiento de la compañía presenta una tendencia de decrecimiento hasta el 2010, mostrando luego un pequeño crecimiento. Esto demuestra que es posible que la empresa haya tenido tendencia a recurrir a fondos internos para la financiación de nuevos proyectos, con el fin de evitar los costos generados por la información asimétrica.
- Como variables independientes se proponen:

\section{Reinversión de utilidades}

Esta cuenta se analiza con el fin de observar si la primera opción que utiliza la empresa como fuente de financiación es la reinversión de utilidades. Los valores correspondientes a la cuenta de reservas para la firma en los tres años en estudio, son: 
Tabla 11. Reservas de la firma estudiada en millones de pesos

\begin{tabular}{|l|c|c|c|c|c|}
\hline VALOR & $\mathbf{2 0 0 7}$ & $\mathbf{2 0 0 8}$ & $\mathbf{2 0 0 9}$ & $\mathbf{2 0 1 0}$ & $\mathbf{2 0 1 1}$ \\
\hline Reservas & 7131.01 & 8833.24 & 10496.29 & 11190.63 & 12737.57 \\
\hline
\end{tabular}

Fuente: estados financieros de la empresa.

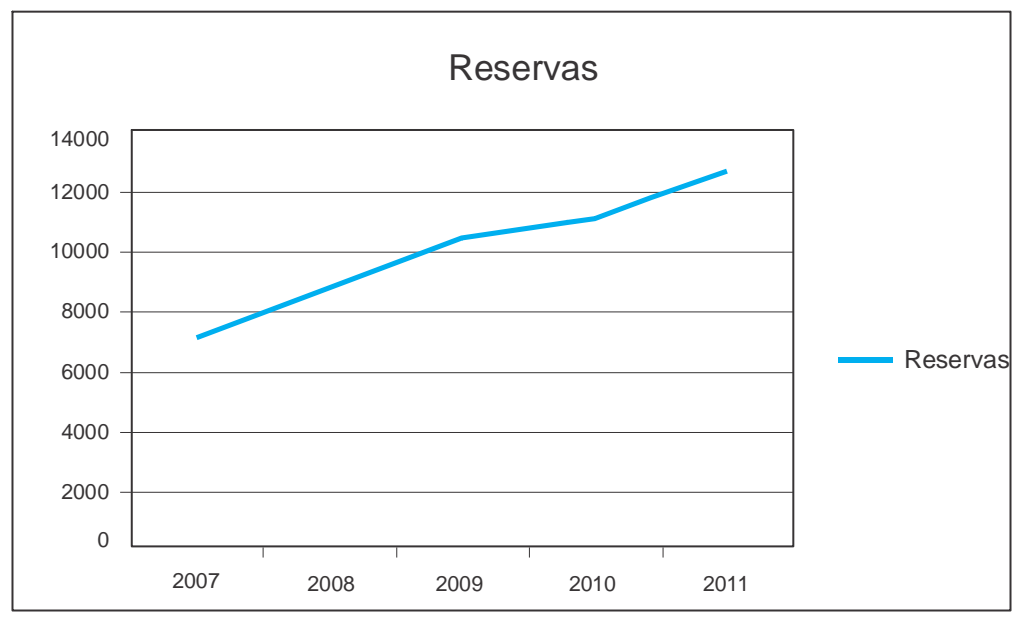

Figura 2. Reservas en millones de pesos.

Fuente: estados financieros de la empresa.

Se observa que durante el año 2008 este monto creció en un $23,8 \%$ con respecto al 2007, y para el 2009 el crecimiento fue del $18,8 \%$ con respecto al 2008. Esto indica que la empresa hizo una apropiación mayor a la reserva legal, por tanto, se puede concluir que utilizó recursos internos para la financiación de nuevas inversiones. Es importante recalcar que la empresa no reparte utilidades a sus socios, pues hace reinversión de sus utilidades en todos los períodos estudiados (Coservicios, 2009).

\section{Crecimiento del AON}

Esta variable es importante ya que supone que en su mayoría el crecimiento de éstos activos operacionales se debe a que la firma debe destinar fondos, ya sean internos o externos para la adquisición de los mismos (ver Tabla 11). 
Teoría del Pecking O rder versusteoría del Trade off para la empresa Coservicios Sandra M ilena Zambrano V argas - Gustavo A dolfo A cuña Corredor

Tabla 12. Resultado de los AON para COSERVICIOS S.A. E.S.P. en millones de pesos

\begin{tabular}{|l|c|c|c|c|}
\hline & $\begin{array}{c}\text { CREC. } \\
\mathbf{2 0 0 7 / 2 0 0 8}\end{array}$ & $\begin{array}{c}\text { CREC. } \\
\mathbf{2 0 0 8} / \mathbf{2 0 0 9}\end{array}$ & $\begin{array}{c}\text { CREC. } \\
\mathbf{2 0 0 9 / 2 0 1 0}\end{array}$ & $\begin{array}{c}\text { DECREC. } \\
\mathbf{2 0 1 0} / \mathbf{2 0 1 1}\end{array}$ \\
\hline$\%$ AON & $17 \%$ & $32.4 \%$ & $16.3 \%$ & $13.6 \%$ \\
\hline
\end{tabular}

Fuente: elaboración de los autores.

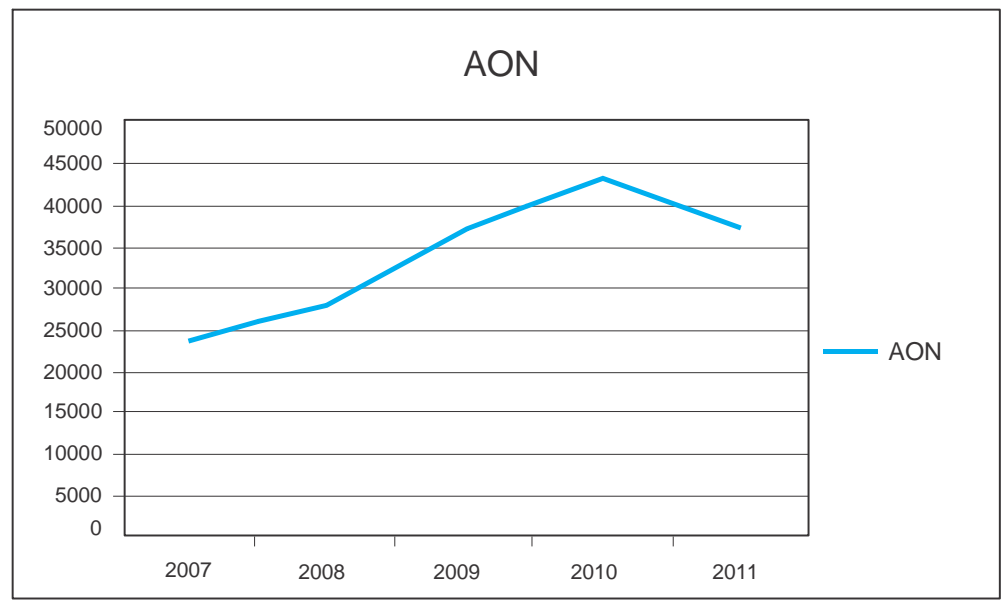

Figura 3. Crecimiento de los activos operacionales netos.

Fuente: elaboración de los autores con base en los estados financieros de la empresa.

En la Figura 3 se observa que hay un crecimiento anual de los activos de operación, siendo del $17 \%$ del 2008 con respecto al 2007 y del $32 \%$ del 2009 con respecto al 2008 y un decrecimiento del 13\% para el 2011 con respecto al 2010. Este último se puede explicar por la adquisición de vehículos a través de leasing. Frank y Goyal (2007) afirman que debe existir una relación negativa entre crecimiento y endeudamiento, de acuerdo con los resultados obtenidos se observa que con una tendencia creciente de los activos operacionales netos, una tendencia creciente en la reinversión de utilidades y unos niveles de endeudamiento decreciente, se puede concluir que la firma recurrió como primera medida a los fondos internos y después pensó en endeudarse en los períodos del 2007 al 2010, lo se puede ver como una aproximación a la jerarquía de preferencias expuesta por Myers (1984), no ocurre lo mismo en el 2011.

Según Myers (1977), citado por Frank y Goyal (2007), las empresas prefieren soportar su apalancamiento en libros (es decir en activos) en lugar de hacerlo en oportunidades de crecimiento. 
Si analizamos el contexto económico y sectorial colombiano, se puede observar que a pesar de la crisis financiera existente en el período de los años 2007 a 2009, los activos de las empresas de servicios públicos crecieron en un $28 \%$ (4,4 billones de pesos), representado principalmente en aquellas que atienden más de 25001 suscriptores. De esas cifras 1,5 billones de pesos corresponden a incrementos en activos fijos, debido a la gran cantidad de inversiones que deben realizarse en éstas empresas para poder operar (Superintendencia de Servicios Públicos, 2010).
La empresa se encuentra en esa misma línea de inversiones y por tanto se respalda el incremento de los activos paralelo a los del sector.

\section{Rentabilidad operativa del activo}

Este índice mide el porcentaje de participación de la utilidad operacional en el total de activos operacionales netos de la firma. Se calcula con la siguiente expresión:

$$
\mathrm{ROA}=\mathrm{UODI} / \mathrm{AON}
$$

Tabla 13. Cálculo de las ROA para COSERVICIOS S.A. E.S.P. en millones de pesos

\begin{tabular}{|l|c|c|c|c|c|}
\hline & $\mathbf{2 0 0 7}$ & $\mathbf{2 0 0 8}$ & $\mathbf{2 0 0 9}$ & $\mathbf{2 0 1 0}$ & $\mathbf{2 0 1 1}$ \\
\hline UODI & 799.11 & 660.45 & 912.65 & 967.71 & 335.31 \\
AON & 24071.36 & 28172.46 & 37305.56 & 43414.73 & 37470.41 \\
ROA & $\mathbf{3 . 3 1 \%}$ & $\mathbf{2 . 3 4 \%}$ & $\mathbf{2 . 4 4 \%}$ & $\mathbf{2 . 2 2 \%}$ & $\mathbf{0 . 8 9 \%}$ \\
\hline
\end{tabular}

Fuente: elaboración de los autores con base en los estados financieros de la empresa.

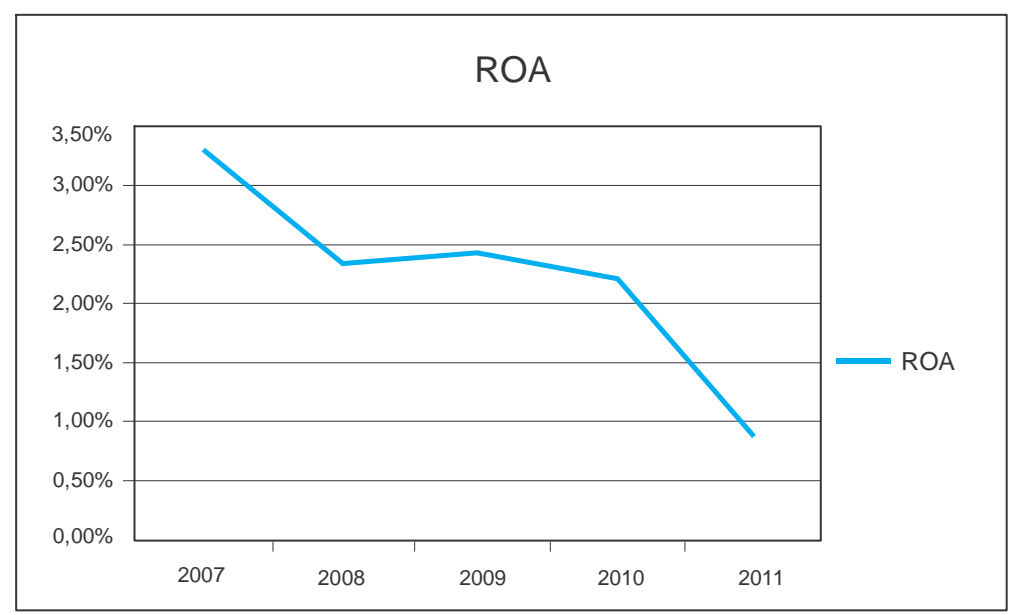

Figura 4. Rentabilidad de los activos operacionales.

Fuente:elaboración de los autores con base en los estados financieros de la empresa. 
Se observa que la rentabilidad de los activos operacionales es baja, lo que indica que la firma tal vez pueda tener un elevado número de activos improductivos que no le están generando utilidad en la operación y que, por el contrario, le disminuye la rentabilidad para los accionistas.

\section{EBITDA}

El EBITDA es un indicador financiero que muestra el resultado de la empresa antes de intereses, impuestos, depreciación, amortización e ítems extraordinarios.

Tabla 14. Cálculo del EBITDA para la empresa en millones de pesos

\begin{tabular}{|l|c|c|c|c|c|}
\hline & $\mathbf{2 0 0 7}$ & $\mathbf{2 0 0 8}$ & $\mathbf{2 0 0 9}$ & $\mathbf{2 0 1 0}$ & $\mathbf{2 0 1 1}$ \\
\hline Utilidad operacional & 1192.7 & 985.75 & 1362.17 & 1444.53 & 500.47 \\
\hline Depreciaciones & 97.4 & 123.01 & 146.57 & 103.42 & 98.33 \\
\hline EBITDA & $\mathbf{1 2 9 0 . 1 1}$ & $\mathbf{1 1 0 8 . 7 6}$ & $\mathbf{1 5 0 8 . 7 4}$ & $\mathbf{1 5 4 7 . 9 5}$ & $\mathbf{5 9 8 . 8 0}$ \\
\hline
\end{tabular}

Fuente: elaboración de los autores con base en los estados financieros de la empresa.

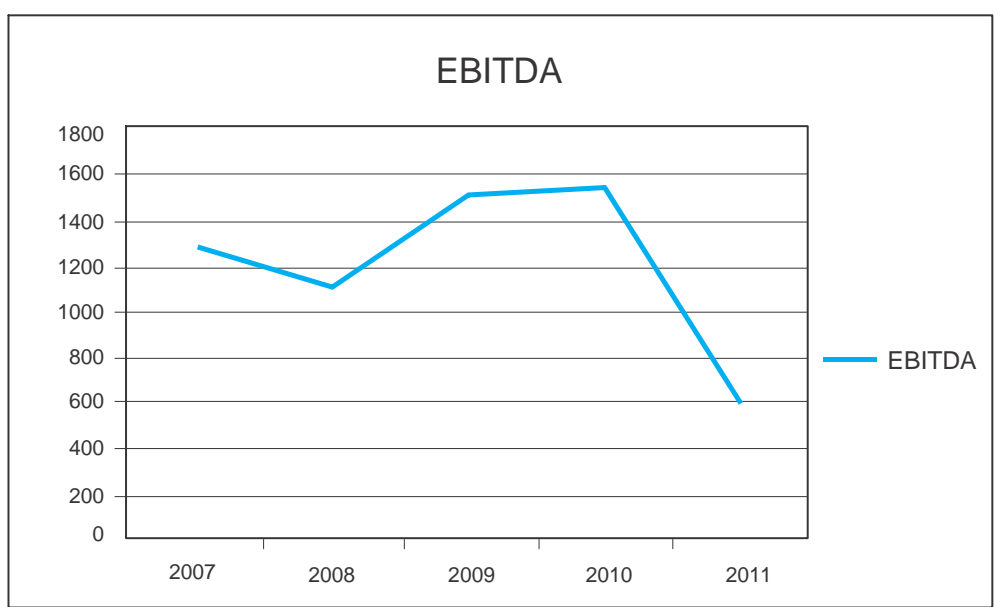

Figura 5.EBITDA para la empresa en estudio.

Fuente: elaboración de los autores con base en los estados financieros de la empresa.

ElEBITDA de la firma estudiada durante los cinco años mostró que su cifra más baja se encontraba en el año 2011, del 2007 al 2008 hubo una disminución del
$14 \%$ que puede analizarse por la crisis del 2008 que redujo considerablemente el consumo de las familias en Colombia. Del año 2008 al 2009 el valor aumentó 
en un $36 \%$, lo que puede explicarse por la tendencia de las empresas en esos años a aumentar sus inversiones y al enorme esfuerzo por reducir costos y hacerse más eficiente (Superintendencia de Servicios Públicos, 2010).

Según la Superintendencia de Servicios Públicos (2010), las empresas de servicios públicos mostraron una dinámica positiva en la generación del EBITDA en el cuatrienio estudiado (incremento del 27\%). Con este aumento, el EBITDA en las empresas del sector contribuyó a mejorar las inversiones en activos sobre todo en lo que respecta a aquellas con más de 25001 suscriptores, como el caso de la organización estudiada.

\section{Análisis de los resultados encontrados de acuerdo con la teoría del Pecking Order}

- La teoría del Pecking Order no considera que exista una estructura óptima de capital en donde se equilibren los beneficios y las desventajas derivados de la deuda. Por el contrario, afirma que las empresas siguen una escala de preferencias hacia el uso interno de recursos para financiar nuevos proyectos de inversión y cuando no le son suficiente recurren a los externos prefiriendo en primer lugar la deuda y finalmente la emisión de acciones (Myers, 1984).

- En cuanto a la relación entre el endeudamiento y la apropiación de reservas, se observa una relación inversamente proporcional. La teoría del Pecking Order explica que en la medida que se utilizan fuentes internas para financiar nuevas inversiones, el endeudamiento debe disminuir (Donaldson, 1961, citado por Myers, 1984) como en efecto sucede en los tres años estudiados.

- Los activos operacionales netos crecieron durante los períodos en estudio, lo contrario ocurrió con el endeudamiento, el cual decreció durante los mismos años. La teoría del Pecking Order establece que ante la necesidad de nuevas inversiones se recurre en primer lugar a los fondos internos. Esto puede validarse con el crecimiento de las reservas y en segundo lugar, con el endeudamiento. Estos resultados muestran que se siguió el orden de jerarquía de preferencias que plantea la teoría en mención, ya que la empresa prefirió usar las reservas antes que endeudarse.

- Según Weston y Copeland (1995), aquellas empresas rentables y con pocas oportunidades de inversión deben tener una razón de endeudamiento baja con relación al capital accionarial, lo contrario ocurre con las que son poco rentables que deberían mostrar unos niveles de endeudamiento altos. Para el presente caso, la rentabilidad operativa del activo se disminuyó en el 2008 y aumentó ligeramente en el 2009. Comparándola con el endeudamiento, que disminuyó en los dos años, podemos decir que no se cumple la 
teoría para el 2008, pues la teoría dice que el endeudamiento disminuye en la medida que aumenta la rentabilidad operativa del activo y en este año la rentabilidad bajó. Para el año 2009, sí se cumplen los lineamientos de la teoría en cuestión. Finalmente, estos resultados se pueden comparar con los hallados por Jaramillo (2009), quien encontró en su estudio que la teoría del Pecking Order se cumplía en una de las empresas estudiadas y no en las otras dos, indicando que existen muchas consideraciones para lograr que se cumpla la teoría y que es muy difícil generalizar su uso y aplicabilidad.

- Según Frank y Goyal (2007) en la teoría del Pecking Order se predice una relación inversa entre apalancamiento y tamaño de la firma. En nuestro caso, por ser una empresa con más de 25001 suscriptores, se considera de tamaño mediano. Analizando esta variable desde la óptica sectorial encontramos que: "Durante el cuatrienio 2006-2009, los prestadores del sector acueducto y alcantarillado tuvieron un continuo desarrollo en su estructura financiera; sin embargo, la mayor evolución se evidenció en las empresas pertenecientes al tamaño con mayor número de suscriptores, lo que mostró en los pequeños prestadores un comportamiento constante durante el período, en el que alcanzan a mantener sus operaciones, aunque sin ganancias adicionales" (Superintendencia de Servicios Públicos, 2010).
- Finalmente, resulta difícil para una empresa regulada recurrir, aun así sea como última opción, a la emisión de acciones, ya que segúnBesankoy Spulbert (1992), existe en las empresas reguladas un problema fundamental de asimetría ya que el precio regulado es flexible pero el capital social de la empresa regulada no lo es. Cuando la empresa regulada ha hecho inversiones irreversibles, el regulador tendría incentivos sólo para cubrir los gastos de funcionamiento pero no para permitirles cierto nivel de rentabilidad a los socios.

\section{CONCLUSIONES}

1. La firma estudiada no se comportó de acuerdo con la teoría del Trade Off, las simulaciones hechas por medio de dos modelos demostraron que los directivos de la empresa en los tres períodos estudiados no se ajustaron gradualmente hacia una estructura óptima de capital. Esto queda validado con los resultados empíricos realizados para el 2007, 2008 y 2009 en donde hubo cierta cercanía entre el valor de endeudamiento óptimo y el valor real. Sin embargo, no hubo solidez en cuanto al modelo propuesto por Cruzet al. (2003), quienes proponen que debe existir armonía entre el valor máximo de las UPA y el valor mínimo del costo de capital en un mismo nivel de endeudamiento. Aquí se presenta la mayor deficiencia para los dos modelos, pues solo se concibe el valor de la empresa como el representado por el 
precio de las UPA, desconociendo aspectos fundamentales a la hora de valorar la firma.

2. Acorde con la teoría del Pecking Order planteada por Myers (1984), que habla de la no existencia de una estructura óptima de capital sino más bien de la existencia de una escala de preferencias, la cual inclina a la firma a escoger la financiación con recursos internos que se hace a través de la reinversión de utilidades, cuando no le son suficientes los recursos, se endeuda y como última opción se inclina por la emisión de acciones. Analizando a la Empresa de Servicios Públicos de Sogamoso desde esta perspectiva, se observa que durante el período comprendido entre los años 2007 a 2009 se le dio prioridad para financiar sus nuevos proyectos de inversión a la generación de recursos internos (visto en los incrementos de la apropiación de reservas), después recurrió al endeudamiento (adquirió vehículos mediante leasing) y no tuvo necesidad de emitir acciones para financiarse, en parte porque dentro de sus políticas actuales no se ha determinado el crecimiento accionario y en parte porque no se emprendieron nuevos proyectos que requirieran grandes inyecciones de capital durante el período en estudio.

3. Se observa a partir del estudio empírico que la Empresa de Servicios Públicos de Sogamoso no siguió la lógica de ninguna de las dos teorías objeto del presente trabajo, por ejemplo, la teoría del Trade Off no se siguió en ninguno de los períodos y la teoría del Pecking Order solo en la primera preferencia en donde la empresa reinvierte sus utilidades (ya que su mayoría accionaria depende del municipio y no hay reparto de utilidades) y en pequeña proporción toma en cuenta el endeudamiento. La última opción nunca se prevé, por tanto la lógica con la que los gerentes han tomado las decisiones financieras en esos años depende más de otras motivaciones como la eficiencia técnica de la compañía y la reglamentación de las tarifas que le permite financiarse de sus propios recursos. Igual que en el estudio de Tenjo et al., (2006) se puede concluir que la existencia de diferentes imperfecciones en el mercado vuelve más complicada la aplicación de las teorías en estudio, pues hace que se tomen diferentes decisiones de endeudamiento dependiendo del acceso a la información financiera. En conclusión y debido al temor por la información asimétrica, las empresas prefieren seguir un orden de jerarquías a la hora de financiarse en lugar de buscar niveles óptimos de deuda.

4. Resulta importante la realización de nuevos estudios en diferentes empresas de la región que permitan llegar a conclusiones más generales acerca de las decisiones de las firmas para su financiación a largo plazo (Sarmiento, 2006). También resulta de 
gran relevancia entender cuáles son los factores determinantes a la hora de decidir el monto de endeudamiento teniendo en cuenta que las empresas pueden tener diferentes motivaciones en el momento de contratar deuda y además puede depender de su tamaño, edad y de diferentes limitaciones en el momento de encontrar fuentes de financiación externa por la asimetría de la información existente.
5. Para López y De Luna (2002), el apalancamiento financiero bien gestionado se convierte en una fuente de creación de valor para una firma. Es por ello que sería importante para los directivos de la empresa en estudio mantener constante la rentabilidad de los activos incrementando el nivel de la deuda dentro de la estructura, financiera siempre y cuando el costo de la deuda sea inferior a la rentabilidad de dichos activos.

\section{R eferencias}

Acuña, G. (2010). El costo de capital de empresas no cotizantes en bolsa. Una aproximación teórica. Ponencia presentada en el II Congreso Internacional de Administración, Investigación -Evolución- Estrategia, realizado en la Universidad Pedagógica y Tecnológica de Colombia, de Tunja, Boyacá.

Averch, H. \& Leland, J. (1962). Behavior of the firm under regulatory cons-traint.The American Economic Review, 52 (85), 1052-1069. Recuperado de: http:// www.jstor.org/stable/1812181

Banco de la República (2010). Informe de la Junta Directiva al Congreso de la república. Recuperado de: http:// www.banrep.gov.co/documentos/ publicaciones/informe_congreso/2010/ marzo.pdf

Banco de la República (2011). Series estadísticas. Recuperado de: http:// www.banrep.gov.co/series-estadisticas/ see_tas_inter.htm

Besanko, D. \& Spulbert, D. (1992).Sequentialequilibrium investment by regulated firms.Rand journal of economics, 23 (2), 153-170. Retrieved from: http:// www.jstor.org/stable/2555981

Bradley, M., Jarrell, G. \& Han Kim, E. (1984). On the Existence of an Optimal Capital Structure: Theory and Evidence. Journal of Finance, 39 (3), 857 878. Retrieved from: http://www.jstor.org/stable/ 2327950

Compañía de Servicios Públicos de Sogamoso COSERVICIOS S.A E.S.P. (2009). Informe de gestión años 2007 y 2008. Estados financieros.

Cruz, J., Villareal, J. \& Rosillo, J. (2003). Finanzas corporativas. Valoración, política de financiamiento y riesgo. Bogotá: Thomson. 
Damodaran Online.(2010, noviembre). Levered and unlevered betas by industry. Retrieved from: http:// pages.stern.nyu.edu/ adamodar/

De Angelo, H. \& Masulis, R. (1980). Optimal capital structure under corporate and personal taxation, Journal of Financial Economics. (8), 3-29. Retrieved from http:/ /papers.ssrn.com/sol3/papers. cfm?abstract_id $=1482270$

Dirección de Impuestos y Aduanas Nacionales -DIAN-. (2007). Ley 1111 de 27 de diciembre de 2006. Recuperado de: http://www.dian.gov.co/dian/ 13Normatividad.nsf/1cffb08b38cf8c 9f05256f88006639f0/6be18f16c6a99b93 052572530056818a?OpenDocument

Empresa de Servicios Públicos de Sogamoso COSERVICIOS S.A E.S.P. Estados financieros de COSERVICIOS S.A E.S.P. para los años 2007, 2008, 2009, $2010 y$ 2011.

Empresa de Servicios Públicos de Sogamoso COSERVICIOS S.AE.S.P. (2009). Informe de Gestión de COSERVICIOS S.A E.S.P. para los años 2007 y 2008.

Frank, M.\&Goyal, V. (2007). Trade-off and Pecking Order Theories of Debt. Hand book of Corporate Finance: Empirical Corporate Finance, 2. Retrieved from http://mba.tuck.dartmouth.edu/pages/ faculty/Es pen. Eckbo/PDFs/ Handbookpdf/CH12-PeckingOrder.pdf

García, O. (2003). Valoración de empresas, gerencia del valor y EVA. (1 ra ed.). Cali: Prensa Moderna Impresores.

Jaramillo, M. (2009). Análisis comparativo de la estructura de capital de tres grandes empresas pertenecientes al subsector de construcción de obras de ingeniería civil, en el período 1998- 2005. Santa $\mathrm{Fe}$ de Bogotá. Tesis para optar por el título de magíster en Administración. Universidad Nacional de Colombia.

Jensen, M. \& Meckling, W. (1976). Theory of the Firm: Managerial Behavior, Agency Costs, and Capital Structure. Journal of Financial Economics, (3), 305 360. Recuperado de: http://hupress. harvard. edu/catalog/JENTHF.html

Kim, E. (1978). A mean-variance theory of optimal capital structure and corporate debt capacity. Journal of Finance, 32(1), 45-63.

Lopez, F. \& De Luna, W. (2002). Finanzas corporativas en la práctica. Madrid: McGraw Hill.

Miller, M. (1977). Debt and taxes. Journal of Finance, 32, 261-275. Retrieved from: http://www.jstor.org/stable/2326758

Modigliani, F. y Miller, M. (1963). "Corporate income taxes and the cost of capital: A correction". American EconomicReview, 53 (3) Recuperado 12/10/2011 de: http:// www.jstor.org/stable/2326758

Moreno, M. (1985). Costes de dificultades financieras y política de endeudamiento empresarial. Revista de Economía y Empresa, 5(12-13), 252-273. Recuperado 05/05/2012 de: http://dialnet. unirioja.es/servlet/articulo?codigo $=2528720$

Myers, S. (1984). Presidential Address: The Capital Structure Puzzle.Journal of Finance, 39(3).575 592. Retrieved from: http://www.jstor.org/stable/2327916. 
Myers, S. \& Majluf, N. (1984). Corporate Financing and Investment Decisions When Firms Have Information that Investors do not Have. Journal of Financial Economics, 13,187 221. Retrieved from http://dspace.mit.edu/ bitstream/handle/1721.1/2068/SWP-1523 15376412.pdf;jsessionid=1C74E8DF96 0E46E73FAFA4DAB8636941

Robichek, A. (1978). Regulation and modern finance theory. The Journal of Finance, 33 (3), 693-705. Retrieved from: http:// www.jstor.org/stable/2326465

Sarmiento, R. (2006). Una aproximación metodológica para determinar la estructura de capital de una firma regulada. Cuadernos Latinoamericanos de Administración, II (1), 33-51. Retrieved from: http://www.uel bosque.edu.co/files/Archivos/file/una aproxmetodo logica.pdf

Shapiro, A. (1991). Establishing a capital structure. In Modern corporate finance. (pp. 458-486). New York: Maxwell MacMillan International.

Shyam, L. \& Myers, S. (1994). Testing Static Trade-off against Pecking Order Models of Capital Structure. MIT Sloan School of Management Cambridge. Working Paper No. 3677. Retrieved from http:// dspace.mit.edu/bitstream/handle/1721.1/ 49224/testingstatictra00shya.pdf? sequence $=1$
Spiegel, Y. \& Spulber, D. (1994). The ca-pital structure of a regulated firm. Rand Journal Economics, 25 (3). Retrieved from: http://www.tau.ac.il/ spiegel/ papers/cap-structure-RJE-1994.pdf

Superintendencia de Servicios Públicos Domiciliarios. (2010). Estudio sectorial acueducto y alcantarillado 2006-2009. Recuperado de: http://www.super servicios.gov.co/c/document_library/ get_file?uuid=89c00aaa-8fe2-479d-b1adba85f5577e52\&groupId=342818

Tenjo, F., López, E. \& Zamudio, N. (2006). Determinantes de la estructura de capital de las empresas colombianas (19962002). Borradores de Economía. Recuperado de: http://www.banrep. gov.co/docum/ftp/borra380.pdf

Warner, J. (1977). Bankruptcycosts: some evidence. The Journal of Finance, 32 (2), 337-347. Retrieved from http:// www.jstor.org/pss/2326766

Weston, J. \& Copeland, T. (1995). Finanzas en administración, tomo II. (Novena ed.). Mexico D.F.: McGraw Hill Interamericana.

Zambrano, S. \& Acuña, G. (2011). Estructura de capital. Evolución teórica. Revista Criterio Libre, 9 (15), 81-102. Recuperado de: http://www.unilibre. edu.co/CriterioLibre/images/revistas/15/ art3.pdf 